\title{
STABILITY OF THE DISCRETIZED PANTOGRAPH DIFFERENTIAL EQUATION
}

\author{
MARTIN BUHMANN AND ARIEH ISERLES
}

\begin{abstract}
In this paper we study discretizations of the general pantograph equation

$$
y^{\prime}(t)=a y(t)+b y(\theta(t))+c y^{\prime}(\phi(t)), \quad t \geq 0, \quad y(0)=y_{0},
$$

where $a, b, c$, and $y_{0}$ are complex numbers and where $\theta$ and $\phi$ are strictly increasing functions on the nonnegative reals with $\theta(0)=\phi(0)=0$ and $\theta(t)<$ $t, \phi(t)<t$ for positive $t$. Our purpose is an analysis of the stability of the numerical solution with trapezoidal rule discretizations, and we will identify conditions on $a, b, c$ and the stepsize which imply that the solution sequence $\left\{y_{n}\right\}_{n=0}^{\infty}$ is bounded or that it tends to zero algebraically, as a negative power of $n$.
\end{abstract}

\section{INTRODUCTION}

In this article we shall consider, in the most general form, the generalized pantograph equation

$$
y^{\prime}(t)=a y(t)+b y(\theta(t))+c y^{\prime}(\phi(t)), \quad t \geq 0, \quad y(0)=y_{0},
$$

where $a, b, c$, and $y_{0}$ are complex and where $\theta$ and $\phi$ are sufficiently smooth "delay" functions on the nonnegative reals which increase strictly monotonically and satisfy $\theta(0)=\phi(0)=0$ and $\theta(t)<t, \phi(t)<t$ for positive $t$. Thus, (1.1) is a differential equation which couples function values and derivatives on distinct time levels. When $c$ vanishes, (1.1) is called a delay equation and the term $c y^{\prime}(\phi(t))$ is called the "neutral" term. Our principal focus in this paper is the case of proportional delays $\theta(t)=q t$ and $\phi(t)=p t$, where $q$ and $p$ are between 0 and 1 . The equation (1.1) can also be considered for vector-valued $y$ and matrices $a, b, c$, but we will dispense with this generalization here.

There are many applications for the generalized pantograph equation. Here we only mention applications in number theory (Mahler [15]), in electrodynamics (Fox et al. [9]) and the collection of current by the pantograph of an electric locomotive (whence its name; cf. Ockendon and Tayler [18]), and in nonlinear dynamical systems (Derfel [6]). A more comprehensive list features in Iserles [12].

Delay differential equations with constant delays, i.e., $c=0$ and $\theta(t)=t-\tau$, where one also prescribes $y$ 's values on $(-\tau, 0)$, have been investigated extensively in the past (see, for instance, Bellman and Cooke [1, 2] and Hale [10]).

Received by the editor October 18, 1991 and, in revised form, May 8, 1992.

1991 Mathematics Subject Classification. Primary 34K20, 65L20; Secondary 34K40. 
However, the analysis of stability of the analytic solution and the numerical solution of a discretized form of (1.1) when the neutral term is present and $\theta(t)=q t$ and $\phi(t)=p t$ (or even with more general forms of $\theta$ and $\phi$ ) has only recently come of age. We mention the paper by the second author [12] concerning the stability of the analytic solution of (1.1), two articles by the authors $[3,4]$ about the stability of numerical solutions when $\theta(t)=\phi(t)=L^{-1} t$, where $L>1$ is an integer, and the reader is referred, for further contributions to the subject, to Carr and Dyson [5], Fox et al. [9], Feldstein and Jackiewicz [8], Kato and McLeod [14], and to Morris, Feldstein, and Bowen [17] for other special cases of (1.1). Stability analysis of the exact solution of (1.1) is the theme of two forthcoming papers, Feldstein et al. [7] and Iserles and Terjéki [13].

In the present paper we shall provide sufficient conditions on $a, b, c$, and the stepsize $h$ so that the solution of (1.1), when discretized according to the trapezoidal rule (there will also be a generalization of this approach in our last section), is bounded or majorized by a sequence that decreases algebraically. When deriving discretizations for differential equations with delay terms it is usual to find values of $y(\theta(t))$ and $y(\phi(t))$ at gridpoints by polynomial interpolation (Meinardus and Nürnberger [16]) unless $\theta(t)$ and $\phi(t)$ happen to be on the grid themselves. This will be an essential ingredient to our approach too. We will study conditions that admit the solution of a trapezoidal rule discretization to be bounded or algebraically decreasing first, and we will identify conditions for these two properties to hold for general $\theta$ and $\phi$ and for the case of two proportional delays $\theta(t)=q t$ and $\phi(t)=p t$, respectively. These are our topics in the following and in the third section. In the fourth section we will give conditions such that the solution vector of a general nonstationary recurrence relation, the trapezoidal rule being a special case thereof, is bounded. The case of proportional delays only will be covered in that part of our work.

\section{BOUNDEDNESS FOR GENERAL MONOTONE DELAYS}

In this section we will derive conditions which imply that the solution sequence of a discretization of (1.1) is bounded. These conditions will depend on $a, b, c$, the stepsize $h$ and the size of the derivatives of $\theta$ and $\phi$ and their inverses. In order to facilitate the presentation of our results, we deal with the case $c=0$, i.e., the "pure delay" case, first. Therefore, suppose that $\theta$ is a differentiable, strictly monotonically increasing function on the nonnegative reals such that $\theta(0)=0$ and $\theta(t)<t$ for all $t>0$, and define $\psi:=\theta^{-1}$. Our present aim is to establish a framework to extend the trapezoidal rule approximation to the solution of (1.1). In order to identify the recurrence relation for our discretization, we integrate (1.1) and obtain

$$
y(t)=y_{0}+a \int_{0}^{t} y(\tau) d \tau+b \int_{0}^{\theta(t)} \psi^{\prime}(\tau) y(\tau) d \tau
$$

This implies that, for all nonnegative integers $n$,

$$
y((n+1) h)=y(n h)+a \int_{n h}^{(n+1) h} y(\tau) d \tau+b \int_{\theta(n h)}^{\theta((n+1) h)} \psi^{\prime}(\tau) y(\tau) d \tau
$$

Let $y_{n}$ denote our approximation to $y(n h)$ for a given stepsize $h$. We dis- 
cretize the first integral in (2.2) with the trapezoidal rule, viz.,

$$
\int_{n h}^{(n+1) h} y(\tau) d \tau \approx \frac{1}{2} h\left(y_{n}+y_{n+1}\right) .
$$

It requires more effort to provide a trapezoidal rule approximation of the second integral in (2.2) as a linear combination of $y_{[\theta(n h) / h]}$ and $y_{[\theta(n h) / h]+j_{n}}$ for some positive $j_{n}$. In order that the recurrence relation becomes explicit for large $n$, we always require that

$$
[\theta(n h) / h]+j_{n}<n+1,
$$

except for $n=0$, when $j_{0}=1$, and perhaps for a further finite number of $n$ 's. In those instances we allow equality instead of the inequality in (2.4). We label the $n$ 's for which (2.4) is an equality as $n_{1} \quad(=0)<n_{2}<\cdots<n_{J}$. We note that (2.4) can always be achieved for $n>0$, since $\theta(t)<t$ for positive $t$. Our strategy is to find the values of $y$ at the endpoints $\theta(n h)$ and $\theta((n+1) h)$ of the range of integration of the second integral in (2.2) by linear interpolation. Hence, it is desirable to have $h[\theta(n h) / h]+j_{n} h$ as near as possible to $\theta((n+1) h)$ without violating (2.4). Since the choice of $j_{n}$ clearly depends on $\theta$, we leave it open how to choose it for general $\theta$, bearing in mind that $j_{n} \equiv 1$ is always possible. We obtain

$$
\int_{\theta(n h)}^{\theta((n+1) h)} \psi^{\prime}(\tau) y(\tau) d \tau \approx \beta_{n} y_{\left[\tilde{\theta}_{n}\right]}+\alpha_{n} y_{\left[\tilde{\theta}_{n}\right]+j_{n}},
$$

where we define

$$
\theta_{n}:=\theta(n h), \quad \tilde{\theta}_{n}:=\frac{\theta_{n}}{h}
$$

and

$$
\begin{array}{r}
\alpha_{n}=j_{n}^{-1}\left(\theta_{n+1}-\theta_{n}\right)\left(\frac{1}{2}\left(\psi^{\prime}\left(\theta_{n}\right) \tilde{\theta}_{n}+\psi^{\prime}\left(\theta_{n+1}\right) \tilde{\theta}_{n+1}\right)\right. \\
\left.-\frac{1}{2}\left(\psi^{\prime}\left(\theta_{n}\right)+\psi^{\prime}\left(\theta_{n+1}\right)\right)\left[\tilde{\theta}_{n}\right]\right)>0 \\
\beta_{n}=\left(\theta_{n+1}-\theta_{n}\right)\left(\frac{1}{2}\left(\psi^{\prime}\left(\theta_{n}\right)+\psi^{\prime}\left(\theta_{n+1}\right)\right)\left(1+j_{n}^{-1}\left[\tilde{\theta}_{n}\right]\right)\right. \\
\left.-\frac{1}{2 j_{n}}\left(\psi^{\prime}\left(\theta_{n}\right) \tilde{\theta}_{n}+\psi^{\prime}\left(\theta_{n+1}\right) \tilde{\theta}_{n+1}\right)\right) .
\end{array}
$$

We obtain the recurrence

$$
y_{n+1}=R y_{n}+S\left(\beta_{n} y_{\left[\tilde{\theta}_{n}\right]}+\alpha_{n} y_{\left[\tilde{\theta}_{n}\right]+j_{n}}\right),
$$

where $R$ and $S$ are the quantities

$$
R=\frac{1+\frac{1}{2} h a}{1-\frac{1}{2} h a} \quad \text { and } \quad S=\frac{b}{1-\frac{1}{2} h a} .
$$

In order that (2.7) always has a nontrivial solution, we require that $S \neq \alpha_{n_{j}}^{-1}$ for $j=1,2, \ldots, J$. We obtain the following result.

Theorem 1. If we denote $\omega=\sup _{n \in \mathbb{Z}_{+}}\left(\alpha_{n}+\left|\beta_{n}\right|\right)$, then $\left\{y_{n}\right\}_{n=0}^{\infty}$ is a bounded sequence if $|R|+\omega|S| \leq 1$. Moreover, the bound

$$
\omega \leq h\left\|\theta^{\prime}\right\|_{\infty}\left\|\psi^{\prime}\right\|_{\infty}\left(1+\left\|\theta^{\prime}\right\|_{\infty}\right)
$$

is true provided that $\left\|\theta^{\prime}\right\|_{\infty}$ and $\left\|\psi^{\prime}\right\|_{\infty}$ are finite. 
Proof. Except for (2.9), the assertion of the theorem immediately follows from (2.7) and from the definition of $\omega$. So we only need to prove (2.9) in order to establish this theorem. Because of $\theta$ 's monotonicity and the mean value theorem, it is true that

$$
0<\theta_{n+1}-\theta_{n} \leq h\left\|\theta^{\prime}\right\|_{\infty} .
$$

We may now obtain a bound on $\omega$ as follows. Employing (2.10), we have

$$
\alpha_{n} \leq h\left\|\theta^{\prime}\right\|_{\infty} \lambda_{n}, \quad\left|\beta_{n}\right| \leq h\left\|\theta^{\prime}\right\|_{\infty}\left|\frac{1}{2}\left(\psi^{\prime}\left(\theta_{n}\right)+\psi^{\prime}\left(\theta_{n+1}\right)\right)-\lambda_{n}\right|,
$$

where

$$
\lambda_{n}:=j_{n}^{-1}\left(\frac{1}{2}\left(\psi^{\prime}\left(\theta_{n}\right) \tilde{\theta}_{n}+\psi^{\prime}\left(\theta_{n+1}\right) \tilde{\theta}_{n+1}\right)-\frac{1}{2}\left(\psi^{\prime}\left(\theta_{n}\right)+\psi^{\prime}\left(\theta_{n+1}\right)\right)\left[\tilde{\theta}_{n}\right]\right),
$$

which is positive. We deduce that

$$
\alpha_{n}+\left|\beta_{n}\right| \leq h\left\|\theta^{\prime}\right\|_{\infty} \max \left(\left\|\psi^{\prime}\right\|_{\infty},\left|2 \lambda_{n}-\frac{1}{2} \psi^{\prime}\left(\theta_{n}\right)-\frac{1}{2} \psi^{\prime}\left(\theta_{n+1}\right)\right|\right) .
$$

Furthermore, using the fact that $\left|\tilde{\theta}_{n}-\left(\left[\tilde{\theta}_{n}\right]+j_{n} / 2\right)\right| \leq j_{n} / 2$, for positive integers $j_{n}$, we see that the second expression in the maximum is at most

$$
\left\|\psi^{\prime}\right\|_{\infty}+j_{n}^{-1}\left|\psi^{\prime}\left(\theta_{n+1}\right) \tilde{\theta}_{n+1}-\psi^{\prime}\left(\theta_{n+1}\right) \tilde{\theta}_{n}\right| \leq\left\|\psi^{\prime}\right\|_{\infty}\left(1+\left\|\theta^{\prime}\right\|_{\infty}\right),
$$

because of the bound (2.10). Hence, we obtain the bound (2.9) for $\omega$ as required.

In view of the left-hand side of (2.11), the influence of the choice of the $j_{n}$ 's may be reflected in the bound on $\omega$ by replacing (2.9) by

$$
\omega \leq h\left\|\theta^{\prime}\right\|_{\infty}\left(\left\|\psi^{\prime}\right\|_{\infty}+\sup _{n \geq 0}\left[j_{n}^{-1}\left|\psi^{\prime}\left(\theta_{n+1}\right)\right| \sup _{t \in[n h,(n+1) h]}\left|\theta^{\prime}(t)\right|\right]\right) .
$$

Corollary 2. The solution sequence of (2.7) is bounded if

$$
|b| \cdot\left\|\theta^{\prime}\right\|_{\infty}\left\|\psi^{\prime}\right\|_{\infty}\left(1+\left\|\theta^{\prime}\right\|_{\infty}\right) \leq \frac{-2 \operatorname{Re} a}{\left|1-\frac{1}{2} h a\right|+\left|1+\frac{1}{2} h a\right|} .
$$

Proof. We need to show that $|R|+\omega|S| \leq 1$ under the condition (2.12). Substituting the values for $R$ and $S$ from (2.8) shows that we need to require

$$
\omega|b| \leq\left|1-\frac{1}{2} h a\right|-\left|1+\frac{1}{2} h a\right| \text {. }
$$

Multiplication of both sides of (2.13) with

$$
\left|1-\frac{1}{2} h a\right|+\left|1+\frac{1}{2} h a\right|
$$

and employing (2.9) yields (2.12).

We shall see later (Theorem 6 in $\S 3$ ) that (2.9) gives a bound which is optimal for the case $\theta(t)=q t$ and irrational $q$, whereas it can be slightly improved for $\theta(t)=q t$ and rational $q$.

We continue the analysis by taking the neutral term into consideration. Now let $\phi$ also be a strictly monotonically increasing function on the nonnegative reals with $\phi(0)=0$ and $\phi(t)<t$ for positive $t$. Let $\phi$ be twice differentiable, 
and denote $\zeta:=\phi^{-1}$. We now integrate (1.1) and employ integration by parts to obtain

$$
\begin{aligned}
y(t)=y_{0} & +a \int_{0}^{t} y(\tau) d \tau+b \int_{0}^{\theta(t)} \psi^{\prime}(\tau) y(\tau) d \tau \\
& +c\left\{y(\phi(t)) \zeta^{\prime}(\phi(t))-y_{0} \zeta^{\prime}(0)-\int_{0}^{\phi(t)} \zeta^{\prime \prime}(\tau) y(\tau) d \tau\right\} .
\end{aligned}
$$

This implies that

$$
\begin{aligned}
y((n+1) h)= & y(n h)+a \int_{n h}^{(n+1) h} y(\tau) d \tau+b \int_{\theta(n h)}^{\theta((n+1) h)} \psi^{\prime}(\tau) y(\tau) d \tau \\
& +c y(\phi((n+1) h)) \zeta^{\prime}(\phi((n+1) h))-c y(\phi(n h)) \zeta^{\prime}(\phi(n h)) \\
& -c \int_{\phi(n h)}^{\phi((n+1) h)} \zeta^{\prime \prime}(\tau) y(\tau) d \tau .
\end{aligned}
$$

We have to supply a trapezoidal rule approximation of the third integral as a linear combination of $y_{[\phi(n h) / h]}$ and $y_{[\phi(n h) / h]+k_{n}}$ for some $k_{n} \geq 1$, where

$$
[\phi(n h) / h]+k_{n}<n+1 \text {, }
$$

except for $n=0$ (when $k_{0}=1$ ) and perhaps for a further finite number of $n$ 's. Again, we then replace the strict inequality by equality in (2.14). We define the set $\left\{\hat{n}_{1}, \hat{n}_{2}, \ldots, \hat{n}_{K}\right\}$ as the set of indices $n$ where $(2.14)$ holds as an equality, $\hat{n}_{1}$ being 0 . The integral is dealt with in the same way as in (2.5), and linear interpolation will also be used to approximate the fourth and fifth terms on the right-hand side. As a result, the expression

$$
\begin{aligned}
\int_{\theta(n h)}^{\theta((n+1) h)} \zeta^{\prime \prime}(\tau) y(\tau) d \tau & -y(\phi((n+1) h)) \zeta^{\prime}(\phi((n+1) h)) \\
& +y(\phi(n h)) \zeta^{\prime}(\phi(n h))
\end{aligned}
$$

is approximated by

$$
\hat{\beta}_{n} y_{\left[\tilde{\phi}_{n}\right]}+\hat{\alpha}_{n} y_{\left[\tilde{\phi}_{n}\right]+k_{n}},
$$

where $\phi_{n}$ and $\tilde{\phi}_{n}$ are defined in a way analogous to the definitions of $\theta_{n}$ and $\tilde{\theta}_{n}$ in (2.6) and where

$$
\begin{aligned}
\hat{\alpha}_{n}= & k_{n}^{-1}\left(\phi_{n+1}-\phi_{n}\right)\left(\frac{1}{2}\left(\zeta^{\prime \prime}\left(\phi_{n}\right) \tilde{\phi}_{n}+\zeta^{\prime \prime}\left(\phi_{n+1}\right) \tilde{\phi}_{n+1}\right)-\frac{1}{2}\left(\zeta^{\prime \prime}\left(\phi_{n}\right)+\zeta^{\prime \prime}\left(\phi_{n+1}\right)\right)\left[\tilde{\phi}_{n}\right]\right) \\
& +k_{n}^{-1}\left(\zeta^{\prime}\left(\phi_{n}\right) \tilde{\phi}_{n}-\zeta^{\prime}\left(\phi_{n+1}\right) \tilde{\phi}_{n+1}\right)+k_{n}^{-1}\left[\tilde{\phi}_{n}\right]\left(\zeta^{\prime}\left(\phi_{n+1}\right)-\zeta^{\prime}\left(\phi_{n}\right)\right), \\
\hat{\beta}_{n}= & \left(\phi_{n+1}-\phi_{n}\right)\left(\frac{1}{2}\left(\zeta^{\prime \prime}\left(\phi_{n}\right)+\zeta^{\prime \prime}\left(\phi_{n+1}\right)\right)\left(1+\frac{1}{k_{n}}\left[\tilde{\phi}_{n}\right]\right)\right. \\
& \left.-\frac{1}{2 k_{n}}\left(\zeta^{\prime \prime}\left(\phi_{n}\right) \tilde{\phi}_{n}+\zeta^{\prime \prime}\left(\phi_{n+1}\right) \tilde{\phi}_{n+1}\right)\right) \\
& -k_{n}^{-1}\left(\zeta^{\prime}\left(\phi_{n}\right) \tilde{\phi}_{n}-\zeta^{\prime}\left(\phi_{n+1}\right) \tilde{\phi}_{n+1}\right)+\left(1+k_{n}^{-1}\left[\tilde{\phi}_{n}\right]\right)\left(\zeta^{\prime}\left(\phi_{n}\right)-\zeta^{\prime}\left(\phi_{n+1}\right)\right) .
\end{aligned}
$$

We obtain the recurrence

$$
y_{n+1}=R y_{n}+S\left(\beta_{n} y_{\left[\tilde{\theta}_{n}\right]}+\alpha_{n} y_{\left[\tilde{\theta}_{n}\right]+j_{n}}\right)-T\left(\hat{\beta}_{n} y_{\left[\tilde{\phi}_{n}\right]}+\hat{\alpha}_{n} y_{\left[\tilde{\phi}_{n}\right]+k_{n}}\right)
$$

where

$$
T=\frac{c}{1-\frac{1}{2} h a}
$$


In order that (2.17) always has a nontrivial solution, we require that $S \neq \alpha_{n_{j}}^{-1}$ for $j=1,2, \ldots, J$ except for the $j$ 's with $n_{j} \in\left\{\hat{n}_{1}, \hat{n}_{2}, \ldots, \hat{n}_{K}\right\}$, and $T \neq$ $-\hat{\alpha}_{\hat{n}_{k}}^{-1}$ for $k=1,2, \ldots, K$ except when $\hat{n}_{k} \in\left\{n_{1}, n_{2}, \ldots, n_{J}\right\}$, and finally $\alpha_{n_{j}} S-\hat{\alpha}_{\hat{n}_{k}} T \neq 1$ for $n_{j}=\hat{n}_{k}$.

We may now state the next result of this section.

Theorem 3. If we denote $\hat{\omega}=\sup _{n \in \mathbb{Z}_{+}}\left(\left|\hat{\alpha}_{n}\right|+\left|\hat{\beta}_{n}\right|\right)$ and recall $\omega$ 's definition from the previous theorem, then $\left\{y_{n}\right\}_{n=0}^{\infty}$ is bounded if $|R|+\omega|S|+\hat{\omega}|T| \leq 1$. Moreover, the estimate

$$
\hat{\omega} \leq h\left\|\phi^{\prime}\right\|_{\infty}\left\|\zeta^{\prime \prime}\right\|_{\infty}\left(3+\left\|\phi^{\prime}\right\|_{\infty}\right)+2\left\|\phi^{\prime}\right\|_{\infty}\left\|\zeta^{\prime}\right\|_{\infty}
$$

is valid provided that $\left\|\phi^{\prime}\right\|_{\infty},\left\|\zeta^{\prime}\right\|_{\infty}$, and $\left\|\zeta^{\prime \prime}\right\|_{\infty}$ are all finite.

Proof. Again, the assertion of the theorem follows from (2.17) and our definitions of $\omega$ and $\hat{\omega}$, except for the bound (2.19). We get this estimate by the same methods as in Theorem 1 and by using the following two estimates:

$$
\begin{aligned}
& k_{n}^{-1} \mid \zeta^{\prime}\left(\phi_{n}\right)\left(\tilde{\phi}_{n}-\left[\tilde{\phi}_{n}\right]\right)-\zeta^{\prime}\left(\phi_{n+1}\right)\left(\tilde{\phi}_{n+1}-\left[\tilde{\phi}_{n}\right]\right) \\
& \quad \leq k_{n}^{-1}\left|\left(\zeta^{\prime}\left(\phi_{n}\right)-\zeta^{\prime}\left(\phi_{n+1}\right)\right)\left(\tilde{\phi}_{n}-\left[\tilde{\phi}_{n}\right]\right)\right|+k_{n}^{-1}\left|\zeta^{\prime}\left(\phi_{n+1}\right)\left(\tilde{\phi}_{n}-\tilde{\phi}_{n+1}\right)\right| \\
& \quad \leq h\left\|\zeta^{\prime \prime}\right\|_{\infty}\left\|\phi^{\prime}\right\|_{\infty}+\left\|\zeta^{\prime}\right\|_{\infty}\left\|\phi^{\prime}\right\|_{\infty}
\end{aligned}
$$

and

$$
\begin{aligned}
& k_{n}^{-1}\left|\zeta^{\prime}\left(\phi_{n}\right)\left(k_{n}+\left[\tilde{\phi}_{n}\right]-\tilde{\phi}_{n}\right)-\zeta^{\prime}\left(\phi_{n+1}\right)\left(k_{n}+\left[\tilde{\phi}_{n}\right]-\tilde{\phi}_{n+1}\right)\right| \\
& \leq h\left\|\zeta^{\prime \prime}\right\|_{\infty}\left\|\phi^{\prime}\right\|_{\infty}+\left\|\zeta^{\prime}\right\|_{\infty}\left\|\phi^{\prime}\right\|_{\infty} .
\end{aligned}
$$

Here we have also used the fact that $\left|k+\left[\tilde{\phi}_{n}\right]-\tilde{\phi}_{n}\right| \leq \max [k, 1]$ for nonnegative integers $k$. The theorem is proved.

Instead of the estimate (2.19), we may bound $\hat{\omega}$ above by

$$
\begin{aligned}
& \hat{\omega} \leq 2 h\left\|\phi^{\prime}\right\|_{\infty}\left\|\zeta^{\prime \prime}\right\|_{\infty} \\
&+\sup _{n \geq 0} {\left[k _ { n } ^ { - 1 } \left(\left\{h\left\|\phi^{\prime}\right\|_{\infty}\left|\zeta^{\prime \prime}\left(\phi_{n+1}\right)\right|+2\left|\zeta^{\prime}\left(\phi_{n+1}\right)\right|\right\}\right.\right.} \\
&\left.\left.\times \sup _{t \in[n h,(n+1) h]}\left|\phi^{\prime}(t)\right|+h \sup _{t \in[n h,(n+1) h]}\left|\zeta^{\prime \prime}(\phi(t)) \phi^{\prime}(t)\right|\right)\right]
\end{aligned}
$$

in order to exhibit the dependence of this bound on $k_{n}, n=1,2, \ldots$.

Corollary 4. The solution sequence of (2.17) is bounded if

$$
\begin{gathered}
|b| \cdot\left\|\theta^{\prime}\right\|_{\infty}\left\|\psi^{\prime}\right\|_{\infty}\left(1+\left\|\theta^{\prime}\right\|_{\infty}\right)+|c| \cdot\left\|\phi^{\prime}\right\|_{\infty}\left\|\zeta^{\prime \prime}\right\|_{\infty}\left(3+\left\|\phi^{\prime}\right\|_{\infty}\right) \\
+2|c| h^{-1}\left\|\phi^{\prime}\right\|_{\infty}\left\|\zeta^{\prime}\right\|_{\infty} \leq \frac{-2 \operatorname{Re} a}{\left|1-\frac{1}{2} h a\right|+\left|1+\frac{1}{2} h a\right|} .
\end{gathered}
$$

We shall see in the following section that the bound (2.19) is attained in the case of proportional delay, i.e., $\phi(t)=p t$.

So far we have chosen to approximate required values of $y$ by linear interpolation at the points where they are needed for the discretization but not available (i.e., for the trapezoidal rule approximation of the second integral in our equation and for the approximation of (2.15)) and to evaluate the delay functions and their derivatives always exactly, because they are available. The 
values of the coefficients $\alpha_{n}, \beta_{n}, \hat{\alpha}_{n}$, and $\hat{\beta}_{n}$ are expressed much more simply, however, if we use linear interpolation to the whole respective integrands and the other terms in (2.15), and therefore we want to record them here too. They are

$$
\begin{aligned}
& \alpha_{n}=j_{n}^{-1}\left(\theta_{n+1}-\theta_{n}\right)\left(\frac{1}{2}\left(\tilde{\theta}_{n}+\tilde{\theta}_{n+1}\right)-\left[\tilde{\theta}_{n}\right]\right) \psi^{\prime}\left(h\left[\tilde{\theta}_{n}\right]+j_{n} h\right)>0, \\
& \beta_{n}=\left(\theta_{n+1}-\theta_{n}\right)\left(1+j_{n}^{-1}\left[\tilde{\theta}_{n}\right]-\frac{1}{2 j_{n}}\left(\tilde{\theta}_{n}+\tilde{\theta}_{n+1}\right)\right) \psi^{\prime}\left(h\left[\tilde{\theta}_{n}\right]\right)
\end{aligned}
$$

and

$\hat{\alpha}_{n}=k_{n}^{-1}\left(\tilde{\phi}_{n+1}-\tilde{\phi}_{n}\right)\left\{\left(\frac{1}{2}\left(\tilde{\phi}_{n}-\tilde{\phi}_{n+1}\right)-\left[\tilde{\phi}_{n}\right]\right) h \zeta^{\prime \prime}\left(h\left[\tilde{\phi}_{n}\right]+k_{n} h\right)-\zeta^{\prime}\left(h\left[\tilde{\phi}_{n}\right]+k_{n} h\right)\right\}$, $\hat{\beta}_{n}=\left(\tilde{\phi}_{n+1}-\tilde{\phi}_{n}\right)\left\{\left(1+k_{n}^{-1}\left[\tilde{\phi}_{n}\right]-\frac{1}{2 k_{n}}\left(\tilde{\phi}_{n}+\tilde{\phi}_{n+1}\right)\right) h \zeta^{\prime \prime}\left(h\left[\tilde{\phi}_{n}\right]\right)+k_{n}^{-1} \zeta^{\prime}\left(h\left[\tilde{\phi}_{n}\right]\right)\right\}$.

Suitable bounds on $\omega$ and $\hat{\omega}$ may now be obtained in the following manner: We note that

$$
\alpha_{n} \leq h\left\|\theta^{\prime}\right\|_{\infty}\left\|\psi^{\prime}\right\|_{\infty} \hat{\lambda}_{n}, \quad\left|\beta_{n}\right| \leq h\left\|\theta^{\prime}\right\|_{\infty}\left\|\psi^{\prime}\right\|_{\infty}\left|1-\hat{\lambda}_{n}\right|,
$$

because of $(2.10)$, where

$$
\hat{\lambda}_{n}:=j_{n}^{-1}\left(\frac{1}{2}\left(\tilde{\theta}_{n}+\tilde{\theta}_{n+1}\right)-\left[\tilde{\theta}_{n}\right]\right),
$$

which is positive. A bound on $\hat{\lambda}_{n}$ is obtained by writing

$$
\hat{\lambda}_{n}=j_{n}^{-1}\left(\tilde{\theta}_{n}-\left[\tilde{\theta}_{n}\right]+\frac{1}{2}\left(\tilde{\theta}_{n+1}-\tilde{\theta}_{n}\right)\right) .
$$

Using the fact that $\tilde{\theta}_{n}-\left[\tilde{\theta}_{n}\right]<1$, we have

$$
\hat{\lambda}_{n} \leq j_{n}^{-1}\left(1+\frac{1}{2} \sup _{t \in[n h,(n+1) h]}\left|\theta^{\prime}(t)\right|\right) \leq j_{n}^{-1}\left(1+\frac{1}{2}\left\|\theta^{\prime}\right\|_{\infty}\right) .
$$

The consequence is the bound

$$
\begin{aligned}
\omega & \leq h\left\|\theta^{\prime}\right\|_{\infty}\left\|\psi^{\prime}\right\|_{\infty} \sup _{n \geq 0}\left[j_{n}^{-1}\left(2+\sup _{t \in[n h,(n+1) h]}\left|\theta^{\prime}(t)\right|-j_{n}\right)\right] \\
& \leq h\left\|\theta^{\prime}\right\|_{\infty}\left\|\psi^{\prime}\right\|_{\infty}\left(1+\left\|\theta^{\prime}\right\|_{\infty}\right) .
\end{aligned}
$$

We get the bound

$$
\begin{gathered}
\widehat{\omega} \leq \sup _{n \geq 0}\left[k _ { n } ^ { - 1 } \left(h\left(2-k_{n}\right)\left\|\phi^{\prime}\right\|_{\infty}\left\|\zeta^{\prime \prime}\right\|_{\infty}+\sup _{t \in[n h,(n+1) h]}\left|\phi^{\prime}(t)\right|\right.\right. \\
\left.\left.\times\left\{h\left\|\phi^{\prime}\right\|_{\infty}\left\|\zeta^{\prime \prime}\right\|_{\infty}+\left|\zeta^{\prime}\left(h\left[\tilde{\phi}_{n}\right]+k_{n} h\right)\right|+\left|\zeta^{\prime}\left(h\left[\tilde{\phi}_{n}\right]\right)\right|\right\}\right)\right] \\
\leq h\left\|\phi^{\prime}\right\|_{\infty}\left\|\zeta^{\prime \prime}\right\|_{\infty}\left(1+\left\|\phi^{\prime}\right\|_{\infty}\right)+2\left\|\phi^{\prime}\right\|_{\infty}\left\|\zeta^{\prime \prime}\right\|_{\infty}
\end{gathered}
$$

in an analogous way.

The analysis of this section allows us to consider, for example, the case

$$
\theta(t)= \begin{cases}q t, & 0 \leq t \leq 1, \\ t-1+q, & t>1,\end{cases}
$$


and, for instance, $\phi(t)=p t$, both $q$ and $p$ being between 0 and 1 . Thus, we have

$$
\begin{aligned}
\theta^{\prime}(t) & = \begin{cases}q, & 0 \leq t \leq 1, \\
1, & t>1,\end{cases} \\
\psi(t) & = \begin{cases}q^{-1} t, & 0 \leq t \leq q, \\
t+1-q, & t>q,\end{cases} \\
\psi^{\prime}(t) & = \begin{cases}q^{-1}, & 0 \leq t \leq q, \\
1, & t>q .\end{cases}
\end{aligned}
$$

It follows that $\left\|\theta^{\prime}\right\|_{\infty}=1$ and $\left\|\psi^{\prime}\right\|_{\infty}=q^{-1}$, and (2.9) supplies a bound on $\omega$ which is similar to the one that occurs when we have proportional delay $\theta(t)=$ $q t$. The present extension is interesting because it combines the proportional delay with constant delay.

We can further generalize the above example by admitting general piecewise linear functions $\theta$ : If $\theta$ is piecewise linear, strictly monotonic and continuous with knots at $0=x_{0}<x_{1}<x_{2}<\cdots$ and values $\theta\left(x_{k}\right)=c_{k}$, where $c_{0}$ is zero, then it is straightforward to see that

$$
\left\|\theta^{\prime}\right\|_{\infty}=\sup _{k>0} \frac{c_{k}-c_{k-1}}{x_{k}-x_{k-1}}
$$

and

$$
\left\|\psi^{\prime}\right\|_{\infty}=\sup _{k>0} \frac{x_{k}-x_{k-1}}{c_{k}-c_{k-1}}
$$

if the knots and function values are such that both of these values are finite. In this fashion, any continuous $\theta$ can be approximated with any accuracy, because the set of piecewise linear functions with free knots is dense in $C\left(\mathbb{R}_{+}\right)$.

\section{STABILITY AND ALgebraic DeCAY FOR PROPORTIONAL DELAYS}

In this section we will identify exactly the constants $\omega$ and $\hat{\omega}$ of the previous section for $\theta(t)=q t$ and $\phi(t)=p t$ in (1.1), thereby sometimes weakening the requirements that lead to bounded sequences and sometimes confirming that the estimates (2.9) and (2.19) on $\omega$ and $\hat{\omega}$ are the best possible. We also obtain a condition that affirms algebraic decay of $\left\{y_{n}\right\}_{n=0}^{\infty}$, i.e., $\left|y_{n}\right|=O\left(n^{\beta}\right)$ for some negative $\beta$, and thus admits the conclusion $\lim _{n \rightarrow \infty} y_{n}=0$. It follows from the work in the previous section that we have the system

$$
\begin{aligned}
y_{0}= & 1, \\
y_{n+1}= & R y_{n}+h S\left(\left(1-\alpha_{n}\right) y_{[q n]}+\alpha_{n} y_{[q n]+1}\right) \\
& +T\left(y_{[p n]+1}-y_{[p n]}\right), \quad n \in \mathbb{Z}_{+},
\end{aligned}
$$

where we took $y(0)=1$ and where $R, S$, and $T$ have been defined in (2.8) and in (2.18), and

$$
\alpha_{n}=\alpha_{n}(q)=q\left(n+\frac{1}{2}\right)-[q n]>0, \quad n=0,1, \ldots
$$

Here we have chosen $j_{n} \equiv k_{n} \equiv 1$ in (2.5) and (2.16). General choices of $j_{n}$ and $k_{n}$ can be accounted for by replacing $\alpha_{n}$ by $\alpha_{n} / j_{n}$ and $T$ by $T / k_{n}$, and $y_{[q n]+1}, y_{[p n]+1}$ by $y_{[q n]+j_{n}}, y_{[p n]+k_{n}}$, respectively, in (3.1), but we will dispense with this straightforward generalization. We require that $1-\frac{1}{2} q h S-T \neq 0$ in order that (3.1) possesses a nontrivial solution. 
In order to state the two principal results of this section, we define

$$
\eta(q):=\sup _{n \in \mathbf{Z}_{+}}\left\{\alpha_{n}+\left|1-\alpha_{n}\right|\right\} .
$$

Note that $\eta(q)=\omega / h$ in view of $\omega$ 's definition in the statement of Theorem 1. Further, $\hat{\omega}=2$ in the present case.

Theorem 5. If

$$
2 h^{-1}|c|+\eta(q)|b| \leq \frac{-2 \operatorname{Re} a}{\left|1-\frac{1}{2} h a\right|+\left|1+\frac{1}{2} h a\right|},
$$

then the solution sequence of (3.1) is bounded. If strict inequality holds in (3.2), then there exists an algebraically decaying majorant to $\left\{y_{n}\right\}_{n=0}^{\infty}$. Specifically, for positive $n$ there is a constant that does not depend on $n$ such that

$$
\left|y_{n}\right| \leq \text { const } \times n^{\log _{\max (1 / p, 1 / q)} A},
$$

where

$$
A=|R|+h \eta(q)|S|+2|T|<1 .
$$

In particular, $\lim _{n \rightarrow \infty} y_{n}=0$.

Theorem 6. The function $\eta(q)$ has the following values:

$$
\eta(q)= \begin{cases}1, & q=K / L \text { and } K \leq 2, \\ 1+q-\frac{2}{L}, & q=K / L \text { and } K \geq 2, \\ 1+q, & q \text { irrational. }\end{cases}
$$

(When $q=K / L$, we assume $K<L$ and $(K, L)$ are relatively prime.)

Theorem 6 shows that the estimate (2.9) on $\omega$ we found in the previous section is best possible for the setting of proportional delays and irrational $q$. We are aware that irrational numbers cannot be represented on a computer, but we believe that it is sensible to consider irrational numbers nevertheless, because in this way we make sure that we know what happens if we are very near an irrational number.

It is perhaps worthwhile to make a comparison between our results above and the results of the authors' earlier paper [3], where we have considered the case $q=L^{-1}, L>1$ an integer. If $c=0$, for instance, it is shown in the latter paper that, when $h$ is small enough, then $\operatorname{Re} a<0$ and $|b|<|a|$ are sufficient for $\lim _{n \rightarrow \infty} y_{n}=0$. Our present requirement (3.2) (with strict inequality) is both worse and better than $\operatorname{Re} a<0,|b|<|a|$ : It is worse, since the set of values of $b$ is considerably smaller, although we note that it is just the right range of values when $a$ is real and negative and $h a>-2$. On the other hand, it is better because it covers the full range of $q \in(0,1)$ and since algebraic decay to zero, which is stronger than $\lim _{n \rightarrow \infty} y_{n}=0$, corresponds to the correct asymptotic behavior of the pantograph equation (Iserles [12]). The roundabout method of proof of Buhmann and Iserles [3], which employs harmonic analysis and ergodic theory, does not provide any useful information on the rate of decay.

Proof of Theorem 5. As far as the boundedness assertion is concerned, we just need to show that $|R|+\eta(q) h|S|+2|T| \leq 1$ under the condition stated in 
the theorem, and this is done in the same way as in Corollary 2. Now to the assertion (3.3): For a given $n>0$, let $m$ be the number of times we can apply the iteration defined through (3.1) without making any of the indices zero. Then, clearly,

$$
\left|y_{n}\right| \leq \text { const } \times(|R|+h \eta(q)|S|+2|T|)^{m+1},
$$

where the constant in (3.5) does not depend on $m$ or $n$. We can find a lower bound on $m$ in the following way. Suppose $p \leq q$; the other case can be treated in the same way. The quantity $[p n]$ is greater than $p n-1$. Therefore, we can find a lower bound on $m$ by looking for the largest $m$ such that

$$
p^{m} n-\sum_{j=1}^{m} p^{j-1}>0 \text {. }
$$

Equivalently, we may require

$$
m+\log _{1 / p} \sum_{j=1}^{m} p^{j-1}<\log _{1 / p} n .
$$

Inequality (3.6) is certainly fulfilled if we get

$$
m+\log _{1 / p} \frac{1}{1-p} \leq \log _{1 / p} n .
$$

Therefore, $m=\log _{1 / p} n+\log _{1 / p}(1-p)$ is an appropriate choice. Hence, the estimate in the statement of the theorem is true.

The proof of Theorem 6 requires more effort; it will occupy the rest of $\S 3$. Our first observation is the following.

Proposition 7. It is true that $\alpha_{n}<1+\frac{1}{2} q$ for all $n \in \mathbb{Z}_{+}$.

Proof. For every $n$ there exists an $l_{n} \in\{0,1, \ldots, n-1\}$ such that

$$
\frac{l_{n}}{n} \leq q<\frac{l_{n}+1}{n} \text {. }
$$

Therefore, we have $l_{n} \leq q n<l_{n}+1$. It follows that $[q n]=l_{n}$, and we thus have

$$
\alpha_{n}=q n+\frac{1}{2} q-l_{n}<\left(l_{n}+1\right)+\frac{1}{2} q-l_{n}=1+\frac{1}{2} q .
$$

This proposition is proved.

Note that, in particular, $\alpha_{n}<\frac{3}{2}$, a bound that can be approached arbitrarily close for some values of $q \in(0,1)$. For instance, we may choose $q=1-\varepsilon$, where $0<\varepsilon \ll 1$. Then we may take $\alpha_{1}=\frac{3}{2}(1-\varepsilon)$.

It follows at once from Proposition 7 and from $\alpha_{n} \geq \frac{1}{2} q$ that

$$
1 \leq \eta(q) \leq 1+q
$$

In particular, we have another proof of (2.9) in the present circumstances. We will now establish the exact expressions asserted in Theorem 6 . In the case of rational $q$ 's we derive

$$
\rho(q):=\sup _{n \in \mathbb{Z}_{+}} \alpha_{n}
$$


(and hence $\eta(q)$ ) explicitly. To this end, let

$$
q=\frac{K}{L},
$$

where $1 \leq K<L$ and $(K, L)$ are relatively prime. Now note that, for any integer $n$,

$$
\frac{n K}{L}-\left[\frac{n K}{L}\right]=\frac{n K \bmod L}{L} .
$$

Since the group $\mathbb{Z} /(L \mathbb{Z})$ is generated by $K$ when $K$ and $L$ are relatively prime ([11]), it follows from (3.8) that

$$
\rho(q)=\frac{1}{2} q+\sup _{n \in \mathbb{Z}}\left(\frac{n K}{L}-\left[\frac{n K}{L}\right]\right)=\frac{1}{2} q+\frac{L-1}{L} .
$$

From this we obtain the following result:

Proposition 8. If $q=K / L$, then

$$
\eta(q)= \begin{cases}1, & K \leq 2, \\ 1+q-\frac{2}{L}, & K \geq 2 .\end{cases}
$$

We now have to deal with the remaining case, namely, irrational $q$. We note that if $q$ is irrational, then the sequence $\{q n-[q n]\}_{n=0}^{\infty}$ is equidistributed in $[0,1]$ (Pólya and Szegö $\left[19\right.$, p. 88]). Hence, for every $\varepsilon>0$ there exists an $n_{\varepsilon}$ such that

$$
1-\varepsilon<q n_{\varepsilon}-\left[q n_{\varepsilon}\right] .
$$

Thus, we get

$$
\alpha_{n_{\varepsilon}}>1+\frac{1}{2} q-\varepsilon
$$

Note that $\varepsilon<\frac{1}{2} q$ implies $\alpha_{n_{\varepsilon}}>1$, and therefore

$$
\eta(q) \geq \alpha_{n_{\varepsilon}}+\left|1-\alpha_{n_{\varepsilon}}\right|=2 \alpha_{n_{\varepsilon}}-1>1+q-2 \varepsilon .
$$

Since $\varepsilon>0$ can be made arbitrarily small, it follows that $\eta(q) \geq 1+q$ and hence, in view of (3.7), also that

$$
\eta(q)=1+q
$$

for irrational $q$. Thus, the proof of Theorem 6 is complete.

We remark that it follows at once from our analysis that $\eta$ is discontinuous everywhere in $(0,1)$. For let $q \in(0,1)$ be rational, $q=K / L$. Then $1+q-$ $\eta(q)=\frac{\min \{K, 2\}}{L}$. Let $q^{*} \in(q-\varepsilon, q+\varepsilon)$ be irrational. Then

$$
\eta\left(q^{*}\right)-\eta(q)>1+q-\eta(q)-\varepsilon=\frac{\min \{K, 2\}}{L}-\varepsilon
$$

and all the irrationals in the neighborhood of $q$ are bounded away from it.

4. BOUNDEDNESS OF THE SOLUTIONS FOR PROPORTIONAL DELAY

In this section, we consider the numerical stability for the more general recurrence relation

$$
y_{n+1}=\sum_{l=0}^{N} r_{l} y_{n-l}+\sum_{l=-M^{\prime}}^{M} \alpha_{l, n} y_{[q n]+l}+\sum_{l=-Q^{\prime}}^{Q} \beta_{l} y_{[p n]+l}
$$


instead of (3.1). Appropriate starting values have to be provided to begin this recursion. Here, $p, q \in(0,1)$, the $r_{l}, \alpha_{l, n}$, and $\beta_{l}$ are complex numbers, and

$$
\alpha_{l, n}=f_{l}(q n-[q n]), \quad l=-M^{\prime},-M^{\prime}+1, \ldots, M, n \in \mathbb{Z}_{+} .
$$

The trapezoidal rule is a special case of (4.1). We require that $[q n]+M$ and $[p n]+Q$ are always at most $n$ for large enough $n, n>\widehat{N}$, say, so that the recursion is explicit for large $n$. We suppose also that $1-\alpha_{l, n}-\beta_{m}$ is nonzero for all $n, l$, and $m$ such that $[q n]+l=[p n]+m=n+1$, that $1-\alpha_{l, n} \neq 0$ for all $n, l$ such that $n+1=[q n]+l$, and finally that $1-\beta_{m} \neq 0$ for all $n$, $m$ such that $n+1=[p n]+m$. We denote

$$
r^{*}:=\sum_{l=0}^{N}\left|r_{l}\right| \quad \text { and } \quad t^{*}:=\sum_{t=-Q^{\prime}}^{Q}\left|\beta_{l}\right| .
$$

Since we have the estimate

$$
\left|y_{n+1}\right| \leq \sum_{l=0}^{N}\left|r_{l}\right|\left|y_{n-l}\right|+\sum_{l=-M^{\prime}}^{M}\left|\alpha_{l, n}\right|\left|y_{[q n]+l}\right|+\sum_{l=-Q^{\prime}}^{Q}\left|\beta_{l}\right|\left|y_{[p n]+l}\right|,
$$

it follows that for $n>\widehat{N}$

$$
\left|y_{n+1}\right| \leq\left(r^{*}+t^{*}+\sum_{l=-M^{\prime}}^{M}\left|\alpha_{l, n}\right|\right) v_{n}
$$

where $v_{n}:=\max _{j=-\max }\left[M^{\prime}, Q^{\prime}\right],-\max \left[M^{\prime}, Q^{\prime}\right]+1, \ldots, n\left|y_{j}\right|$. From this we obtain for $n>\widehat{N}$

$$
v_{n+1} \leq \max \left\{1, r^{*}+t^{*}+\sum_{l=-M^{\prime}}^{M}\left|\alpha_{l, n}\right|\right\} v_{n} .
$$

Therefore, numerical stability, i.e., boundedness of the $y_{n}$ 's, is provided if we can ensure

$$
\prod_{j=0}^{n-1}\left(r^{*}+t^{*}+\sum_{l=-M^{\prime}}^{M}\left|\alpha_{l, j}\right|\right) \leq 1, \quad n \in \mathbb{Z}_{+},
$$

or equivalently,

$$
\frac{1}{n} \sum_{j=0}^{n-1} \log \left(r^{*}+t^{*}+\sum_{l=-M^{\prime}}^{M}\left|\alpha_{l, j}\right|\right) \leq 0 .
$$

If $q$ is irrational, then the equidistribution theorem which we have already used in $\S 3$ implies that

$$
\begin{aligned}
& \frac{1}{n} \sum_{j=0}^{n-1}\left(r^{*}+t^{*}+\sum_{l=-M^{\prime}}^{M}\left|\alpha_{l, j}\right|\right) \\
& \quad=\int_{0}^{1} \log \left(r^{*}+t^{*}+\sum_{l=-M^{\prime}}^{M}\left|f_{l}(t)\right|\right) d t+o(1), \quad n \rightarrow \infty .
\end{aligned}
$$


Thus, we obtain the uniform boundedness condition

$$
\int_{0}^{1} \log \left(r^{*}+t^{*}+\sum_{l=-M^{\prime}}^{M}\left|f_{l}(t)\right|\right) d t<0,
$$

which is sufficient because (4.5) and (4.4) imply (4.3) for large enough $n$. When restricted to the cases studied in the previous section for irrational $q$, we obtain the bound

$$
\begin{aligned}
& (|R|+h|S|+2|T|)^{-|R|+(1-q) h|S|-2|T|}(|R|+(1+q) h|S|+2|T|)^{|R|+(1+q) h|S|+2|T|} \\
& \quad<e^{q h|S|}
\end{aligned}
$$

as a sufficient condition for stability.

If $q$ is rational, viz., $q=\frac{K}{L}$, we exploit the following result:

Proposition 9. Denote $q n-[q n]$ by $\tilde{\alpha}_{n}$. Then for every $m \in\{0,1, \ldots, L-1\}$ and $\tilde{N} \in \mathbb{Z}_{+}$there exists a unique $l \in\{0,1, \ldots, L-1\}$ such that

$$
\tilde{\alpha}_{\widetilde{N} L+l}=\frac{m}{L} \text {. }
$$

Moreover, $l$ is independent of $\tilde{N}$.

Proof. Expression (4.7) is equivalent to

$$
\frac{K}{L}(\tilde{N} L+l)-\left[\frac{K}{L}(\tilde{N} L+l)\right]=\frac{m}{L},
$$

which, in turn, is equivalent to

$$
g(l):=K l-L\left[\frac{K l}{L}\right]=m .
$$

Note that $\tilde{N}$ has disappeared altogether.

Let us suppose that $l_{1}, l_{2} \in\{0,1, \ldots, L-1\}, l_{2}>l_{1}$, exist such that $g\left(l_{1}\right)=g\left(l_{2}\right)$. This is the same as

$$
K\left(l_{2}-l_{1}\right)=L\left(\left[\frac{K l_{2}}{L}\right]-\left[\frac{K l_{1}}{L}\right]\right) .
$$

Let $K l_{j}=d_{j} L+c_{j}, c_{j} \in\{0,1, \ldots, L-1\}, j=1,2$. Then (4.8) implies $c_{1}=c_{2}$, and hence $K\left(l_{2}-l_{1}\right)=L\left(d_{2}-d_{1}\right)$. We obtain

$$
\frac{K}{L}=\frac{d_{2}-d_{1}}{l_{2}-l_{1}},
$$

and this contradicts the requirement that $(K, L)$ are relatively prime (recall that $\left.1 \leq l_{2}-l_{1} \leq L-1\right)$. We deduce that the set $\{g(0), g(1), \ldots, g(L-1)\}$ consists of distinct numbers. Thus, since $g(j) \in\{0,1, \ldots, L-1\}$, it follows that the set is a permutation of $\{0,1, \ldots, L-1\}$ (in other words, $g$ is a bijection of the cyclic group $\left.G_{L-1}\right)$. In particular, there exists $l$ such that $g(l)=m$. 
We have proved that

$$
\left\{\tilde{\alpha}_{n}\right\}_{n=N L}^{N L+L-1}=\text { permutation of }\left\{\frac{j}{L}\right\}_{j=0}^{L-1} .
$$

Hence, we may deduce that a sufficient condition for uniform boundedness of $\left\{y_{n}\right\}_{n=0}^{\infty}$ is

$$
\prod_{j=0}^{L-1}\left(r^{*}+t^{*}+\sum_{l=-M^{\prime}}^{M}\left|f_{l}\left(\frac{j}{L}\right)\right|\right) \leq 1
$$

or equivalently, by taking logarithms,

$$
\frac{1}{L} \sum_{j=0}^{L-1} \log \left(r^{*}+t^{*}+\sum_{l=-M^{\prime}}^{M}\left|f_{l}\left(\frac{j}{L}\right)\right|\right) \leq 0 \text {. }
$$

Thus, we remark that expression (4.5) can be considered as a limiting case of (4.10): if $K_{i} / L_{i} \rightarrow q \notin \mathbb{Q}(i \rightarrow \infty)$ then, clearly, $L_{i} \rightarrow \infty(i \rightarrow \infty)$ and (4.10) becomes the trapezoidal rule approximant of (4.5). (If the $f_{l}$ 's are at least $C^{2}$, the convergence of the trapezoidal rule is assured by the Peano kernel theorem.) We sum up our observations in the following theorem:

Theorem 10. The solution sequence to the recurrence relation (4.1) is bounded if (4.5) or (4.10) hold, depending on whether $q$ is irrational or rational.

Finally, we take a look at the case of rational $q$ in the context of $\S 3$. Then (4.9) reads

$$
\prod_{j=0}^{L-1}\left(|R|+\tilde{\rho}_{j} h|S|+2|T|\right) \leq 1
$$

where

$$
\tilde{\rho}_{j}=\frac{K+2 j}{2 L}+\left|1-\frac{K+2 j}{2 L}\right|, \quad j \in\{0,1, \ldots, L-1\} .
$$

Note that if $j \leq L-\left[\frac{1}{2}(K+1)\right]$, then $\tilde{\rho}_{j}=1$; otherwise $\tilde{\rho}_{j}=(K+2 j-L) / L$. It follows that $(4.11)$ is the same as

$$
(|R|+h|S|+2|T|)^{L} \prod_{j=1}^{[(K-1) / 2]}\left(1+\frac{K-2 j}{L} \frac{h|S|}{|R|+h|S|+2|T|}\right) \leq 1 .
$$

\section{ACKNOWLEDGMENT}

It is a pleasure to thank an anonymous referee for a suggestion which helped to shorten an unnecessarily prolix proof of Proposition 8.

\section{BIBLIOGRAPHY}

1. R. Bellman and K. L. Cooke, Asymptotic behavior of solutions of differential-difference equations, Mem. Amer. Math. Soc. No. 35 (1959).

2. __ Differential-difference equations, Academic Press, New York, 1963.

3. M. D. Buhmann and A. Iserles, On the dynamics of a discretized neutral equation, IMA J. Numer. Anal. 12 (1992), 339-363. 
4. __ Numerical analysis of functional equations with a variable delay, Numerical Analysis 1991 (D. F. Griffiths and G. A. Watson, eds.), Longman, Harlow, 1992, pp. 17-33.

5. J. Carr and J. Dyson, The functional differential equation $y^{\prime}(x)=a y(\lambda x)+b y(x)$, Proc. Roy. Soc. Edinburgh Sect. A 74 (1974-75), 165-174.

6. G. A. Derfel, Kato problem for functional-differential equations and difference Schrödinger operators, Operator Theory 46 (1990), 319-321.

7. A. Feldstein, A. Iserles, and D. Levin, Embedding of delay equations into an infinite-dimensional ODE system, Technical Report DAMTP NA21, University of Cambridge, 1991.

8. A. Feldstein and Z. Jackiewicz, Unstable neutral functional differential equations, Arizona State University Technical Report (1989).

9. L. Fox, D. F. Mayers, J. R. Ockendon, and A. B. Tayler, On a function differential equation, IMA J. Appl. Math. 8 (1971), 271-307.

10. J. Hale, Theory of functional differential equations, Springer-Verlag, New York, 1977.

11. G. H. Hardy and E. M. Wright, An introduction to the theory of numbers, Clarendon Press, Oxford, 1954.

12. A. Iserles, On the generalized pantograph functional-differential equation, European J. Appl. Math. (to appear).

13. A. Iserles and J. Terjéki, Stability and asymptotic stability of functional-differential equations, Technical Report DAMTP NA1, University of Cambridge, 1992.

14. T. Kato and J. B. McLeod, The functional-differential equation $y^{\prime}(x)=a y(\lambda x)+b y(x)$, Bull. Amer. Math. Soc. 77 (1971), 891-937.

15. K. Mahler, On a special functional equation, J. London Math. Soc. 15 (1940), 115-123.

16. G. Meinardus and G. Nürnberger, Approximation theory and numerical methods for delay equations, Delay Equations, Approximation and Application (G. Meinardus and G. Nürnberger, eds.), Birkhäuser-Verlag, Basel, 1986.

17. G. R. Morris, A. Feldstein, and E. W. Bowen, The Phragmén-Lindelöf principle and a class of functional-differential equations, Ordinary Differential Equations (L. Weiss, ed.), Academic Press, New York, 1972.

18. J. R. Ockendon and A. B. Tayler, The dynamics of a current collection system for an electric locomotive, Proc. Roy. Soc. London Ser. A 322 (1971), 447-468.

19. G. Pólya and G. Szegö, Problems and theorems in analysis. Vol. 1, Springer-Verlag, Berlin and New York, 1972.

Magdalene College, Cambridge CB3 0AG, England, and ibM Thomas J. Watson ReSearch Center, P. O. Box 218, Yorktown Heights, New York 10598

E-mail address: mdb@amtp.cam.ac.uk

Department of Applied Mathematics and Theoretical Physics, University of Cambridge, Silver Street, Cambridge CB3 9EW, England

E-mail address: ai@amtp.cam.ac.uk 